\section{Basalzellkrebs an Ohren aggressiver}

Basalzellkarzinome an den Ohren wachsen meist aggressiv und treten häufiger bei Männern auf. Schuld daran ist laut US-Dermatologen die Haarmode.

— ür ihre Analyse griffen Abel Jarell - und Thaddeus Mully von der University of California in San Francisco auf Daten der dortigen dermatologischen Klinik zurück. Sie verglichen die ersten 100 Fälle von Basalzellkrebs am Ohr, die im Jahr 2009 diagnostiziert worden waren, mit den ersten 100 Fällen dieser Krebsart auf der Wange. $57 \%$ der am Ohr lokalisierten Basaliome wiesen einen Hochrisikotyp (infiltrativ, mikronodulär, morpheiform) auf, an der Wange nur $38 \%$ (Odds Ratio [OR] 2,16). 79\% der Ohrbasaliome wurden bei Männern festgestellt (OR 3,34).

UV-Strahlung stellt den wesentlichen Risikofaktor für Basalzellkrebs dar. Entsprechend viele Tumoren sind an Kopf und Nacken zu finden. Die große Geschlechterdifferenz im Auftreten an den Ohren erklären Jarell und Mully mit der Haarmode: Frauen tragen häufiger längeres Haar als Männer, ihre Ohren sind daher vor Sonnenlicht besser geschützt.

Fazit: Am Ohr ist es schwierig zu operieren, und es ist nicht einfach, die lokale Kontrolle über ein dort wachsendes Basaliom zu erlangen. Die Therapie ist sorgfältig zu planen, dafür muss Klarheit über den Tumortyp herrschen. Praktisch wichtig ist daher der Hinweis, dass oberflächliche Biopsien aggressives Wachstum oft nicht erkennen lassen. „Es ist ratsam“, so Jarell und Mully, „bei Verdacht auf einen nicht-melanomatösen

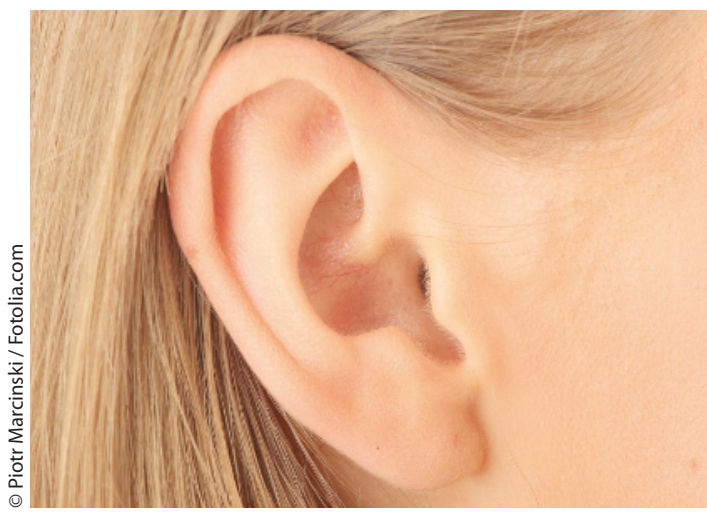

Modische Krebsprophylaxe: Lange Haare schützen die Ohren vor UV-Licht.

Hautkrebs am Ohr Gewebe aus der Tiefe zu gewinnen."

Dr. Robert Bublak

Jarell AD, Mully TW. Basal cell carcinoma on the ear is more likely to be of an aggressive phenotype in both men and women. J Am Acad Dermatol. 2012; 66:780-4

\section{Chronische Rhinosinusitis beim Kind: Mit oralen Glukokortikoiden bekämpfen?}

\begin{abstract}
Topische Glukokortikoide lindern - ergänzend zur Antibiotikatherapie - die nasalen Symptome der chronischen Rhinosinusitis. Ob auch systemische Glukokortikoide für die Behandlung von Kindern mit chronischer Rhinosinusitis geeignet sind, wollten Wissenschaftler jetzt herausfinden.
\end{abstract}

A n einer randomisierten, placebokontrollierten Studie aus der Türkei nahmen 48 Kinder im Alter von sechs bis 17 Jahren mit klinisch und radiologisch bestätigter chronischer Rhinosinusitis und ohne Nasenpolypen teil. Sie erhielten 30 Tage lang zweimal täglich ein Antibiotikum (Amoxicillin/Clavulansäure) zusammen mit Methylprednisolon (oral) oder mit Placebo. Die Wirksamkeit, der primäre Studienendpunkt, wurde anhand subjektiver (VAS-Symptomscore) und objektiver Parameter (CT-Scans der Nasennebenhöhlen) ermittelt. Rückfallraten und Verträglichkeit waren sekundäre Endpunkte.

In beiden Gruppen schied ein Kind wegen Abweichungen vom Protokoll aus. Unabhängig von der Therapie besserten sich Symptome und CT-Befunde signifikant gegenüber dem Ausgangszustand. Methylprednisolon war jedoch wirksamer als Placebo in Bezug auf die Symptome insgesamt, nasale Obstruktion, "postnasal drip“ und Husten. Hinsichtlich Nasensekret, Halitosis und Kopf- oder Gesichtsschmerzen brachte das Glukokortikoid dagegen

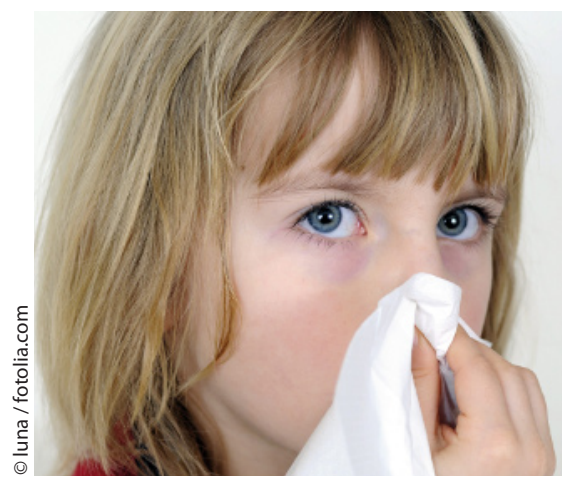

Methylprednisolon bewährt sich als Ergänzung zu Antibiotika bei chronischer Rhinosinusitis im Kindesalter. keinen Vorteil. Vollständige Beschwerdefreiheit oder eine klinisch signifikante Besserung erreichten 17 Glukokortikoid- und sieben Placebopatienten. Die CT-Scores verringerten sich in der GlukokortikoidGruppe ebenfalls signifikant stärker als in der Placebogruppe. 19 von 22 Patienten hatten bei Studienende unauffällige CTScans (Lund-Mackay-Score $\leq 2$ ), im Vergleich zu zwölf von 23 Placebopatienten.

Klinisch relevante Nebenwirkungen der Glukokortikoidtherapie wurden nicht berichtet. Rezidive in den sechs Monaten nach Behandlung traten in der Glukokortikoidgruppe zwar seltener auf (25\% vs. $43 \%$ ), der Unterschied war aber nicht signifikant.

Fazit: Subjektive und objektive Zeichen einer chronischen Rhinosinusitis bei Kindern lassen sich durch Antibiotika plus orales Methylprednisolon besser unter Kontrolle bringen als mit Antibiotika allein.

Dr. Beate Schumacher

Ozturk F et al. Efficacy and tolerability of systemic methylprednisolone in children and adolescents with chronic rhinosinusitis: a double-blind, placebo-controlled randomized trial. J Allergy Clin Immunol 2011; 128 : 348-52 